\title{
Li Diffusion in $\mathrm{Li}_{x} \mathrm{CoO}_{2}$ Probed by Muon-Spin Spectroscopy
}

\author{
Jun Sugiyama, ${ }^{1, *}$ Kazuhiko Mukai, ${ }^{1}$ Yutaka Ikedo, ${ }^{1, \dagger}$ Hiroshi Nozaki, ${ }^{1}$ Martin Månsson, ${ }^{2}$ and Isao Watanabe ${ }^{3}$ \\ ${ }^{1}$ Toyota Central Research and Development Laboratories Inc., Nagakute, Aichi 480-1192 Japan \\ ${ }^{2}$ Laboratory for Neutron Scattering, ETH Zürich and Paul Scherrer Institut, CH-5232 Villigen PSI, Switzerland \\ ${ }^{3}$ Muon Science Laboratory, RIKEN, 2-1 Hirosawa, Wako, Saitama 351-0198, Japan
}

(Received 16 June 2009; published 30 September 2009)

\begin{abstract}
The diffusion coefficient of $\mathrm{Li}^{+}$ions $\left(D_{\mathrm{Li}}\right)$ in the battery material $\mathrm{Li}_{x} \mathrm{CoO}_{2}$ has been investigated by muon-spin relaxation $\left(\mu^{+} \mathrm{SR}\right)$. Based on experiments in zero and weak longitudinal fields at temperatures up to $400 \mathrm{~K}$, we determined the fluctuation rate $(\nu)$ of the fields on the muons due to their interaction with the nuclear moments. Combined with susceptibility data and electrostatic potential calculations, clear $\mathrm{Li}^{+}$ ion diffusion was detected above $\sim 150 \mathrm{~K}$. The $D_{\mathrm{Li}}$ estimated from $\nu$ was in very good agreement with predictions from first-principles calculations, and we present the $\mu^{+}$SR technique as an optimal probe to detect $D_{\mathrm{Li}}$ for materials containing magnetic ions.
\end{abstract}

DOI: 10.1103/PhysRevLett.103.147601

In spite of a long research history on lithium insertion materials for Li-ion batteries [1], e.g., $\mathrm{LiCoO}_{2}, \mathrm{LiNiO}_{2}$, and $\mathrm{LiMn}_{2} \mathrm{O}_{4}$, one of their most important intrinsic physical properties, the $\mathrm{Li}^{+}$ions diffusion coefficient $\left(D_{\mathrm{Li}}\right)$, has not yet been determined with any reliability. Although Li NMR is a powerful technique to measure $D_{\mathrm{Li}}$ for nonmagnetic materials, it is particularly difficult to evaluate $D_{\mathrm{Li}}$ for materials containing magnetic ions, because the magnetic ions induce additional pathways for the spin-lattice relaxation rate $\left(1 / T_{1}\right)$, resulting in huge $1 / T_{1}$ compared with that expected for only the diffusive motion of $\mathrm{Li}$ ions.

Such difficulty was clearly evident in the $1 / T_{1}(T)$ curve for $\mathrm{LiCoO}_{2}$ and $\mathrm{LiNiO}_{2}$ [2,3], and, for that reason, $D_{\mathrm{Li}}$ was instead estimated from the $\mathrm{Li}$ NMR linewidth [4]. However, since the linewidth, i.e., the spin-spin relaxation rate $\left(1 / T_{2}\right)$, is also affected by the magnetic ions, the $D_{\mathrm{Li}}$ obtained by $\mathrm{Li} \mathrm{NMR}$ for $\mathrm{LiCoO}_{2}\left(=1 \times 10^{-14} \mathrm{~cm}^{2} \mathrm{~s}^{-1}\right.$ at $400 \mathrm{~K}$ ) is approximately 4 orders of magnitude smaller than predicted by first-principles calculations [5]. Since lithium insertion materials always include transition metal ions, in order to maintain charge neutrality during the extraction and/or insertion of $\mathrm{Li}^{+}$ions, it is consequently very difficult to determine $D_{\mathrm{Li}}$ for these compounds unambiguously by Li NMR.

On the other hand, the chemical diffusion coefficient $\left(D_{\mathrm{Li}}^{\text {chem }}\right)$, which is measured under a potential gradient, has also been determined by electrochemical measurements. Note that the relationship between $D_{\mathrm{Li}}$ and $D_{\mathrm{Li}}^{\text {chem }}$ is given by $D_{\mathrm{Li}}^{\text {chem }}=\Theta D_{\mathrm{Li}}$, where $\Theta$ is a thermodynamic factor. The magnitude of $D_{\mathrm{Li}}^{\text {chem }}$ is, however, known to be very sensitive to the measurement system, e.g., the electrolyte as well as the compositions of the positive and negative electrodes. As a result, the reported $D_{\mathrm{Li}}^{\text {chem }}$ for $\mathrm{Li}_{x} \mathrm{CoO}_{2}$ ranges from $4 \times 10^{-8}$ to $10^{-10} \mathrm{~cm}^{2} \mathrm{~s}^{-1}$ for powder samples [610] and from $2.5 \times 10^{-11}$ to $2 \times 10^{-13} \mathrm{~cm}^{2} \mathrm{~s}^{-1}$ for thin films [11-14] at ambient $T$. In order to profoundly understand the physics behind the operation principle of battery materials, it is imperative to have a reliable probe to
PACS numbers: 76.75.+i, 66.30.H-, 82.47.Aa, 82.56.Lz

measure $D_{\mathrm{Li}}$ for all the components of the battery as a function of both Li content as well as $T$. This is at present a key issue for further development of Li-ion batteries, and, in particular, for future fabrication of solid-state batteries.

In contrast to NMR, the effect of localized moments in a paramagnetic (PM) state on the muon-spin relaxation $\left(\mu^{+} \mathrm{SR}\right)$ signal is very limited at high $T$, because the PM fluctuation is usually too fast to be visible by $\mu^{+}$SR. Furthermore, although $\mu^{+} \mathrm{SR}$ is very sensitive to the local magnetic environment, whether it is due to electronic or nuclear spins, an electronic contribution is, in principle, distinguishable from a nuclear contribution by weak longitudinal field measurements. In addition, since the $\mu^{+}$SR time scale is different from the NMR's time scale, $\mu^{+} \mathrm{SR}$ is expected to provide unique information on nuclear magnetic fields. Indeed, $\mu^{+} \mathrm{SR}$ studies of $\mathrm{Li}_{x} \mathrm{CoO}_{2}$ at low $T$ indicate that $\mu^{+}$'s feel a nuclear magnetic field caused by $\mathrm{Li}$ and ${ }^{59} \mathrm{Co}$ even at $1.8 \mathrm{~K}[15,16]$. This offers a possibility to determine $D_{\mathrm{Li}}$ of $\mathrm{Li}_{x} \mathrm{CoO}_{2}$ by $\mu^{+} \mathrm{SR}$, if $\mu^{+}$'s locate in the vicinity of the $\mathrm{O}^{2-}$ ion and make a stable $\mu^{+}-\mathrm{O}^{2-}$ bond in the $\mathrm{Li}_{x} \mathrm{CoO}_{2}$ lattice. Here, we report our initial work on $\mathrm{Li}_{x} \mathrm{CoO}_{2}(x=0.73$ and 0.53$)$ to estimate their $D_{\mathrm{Li}}$ and establish $\mu^{+} \mathrm{SR}$ as a novel tool to probe Li-ion diffusion.

A powder sample of $\mathrm{LiCoO}_{2}$ was prepared at Osaka City University by a solid-state reaction technique using reagent grade $\mathrm{LiOH} \cdot \mathrm{H}_{2} \mathrm{O}$ and $\mathrm{CoCO}_{3}$ powders as starting materials. A mixture of the two powders was heated at $900{ }^{\circ} \mathrm{C}$ for $12 \mathrm{~h}$ in air. Powder $\mathrm{x}$-ray diffraction (XRD) analysis showed that the $\mathrm{LiCoO}_{2}$ sample was single phase with a rhombohedral system of space group $R \overline{3} m\left(a_{\mathrm{H}}=\right.$ $0.2814 \mathrm{~nm}$ and $c_{\mathrm{H}}=1.4049 \mathrm{~nm}$ in hexagonal setting). The Li-deficient samples were prepared by an electrochemical reaction using $\mathrm{Li} \mid \mathrm{LiPF}_{6}$-ethylene carbonatediethyl carbonate $\mid \mathrm{LiCoO}_{2}$ cells. The $\mathrm{LiCoO}_{2}$ powder was pressed into a disk with $15 \mathrm{~mm}$ diameter and $0.4 \mathrm{~mm}$ thickness, and the disk was then used as a positive electrode. The $\mathrm{Li}_{x} \mathrm{CoO}_{2}$ disk was removed from the cell in a glovebox and packed into a sealed powder cell just before 
the $\mu^{+} \mathrm{SR}$ measurement. Their structures were subsequently confirmed by powder XRD, and, finally, their compositions were checked by an inductively coupled plasma atomic emission spectral analysis. The above procedure is essentially the same as that of our previous $\mu^{+} \mathrm{SR}$ work on $\mathrm{Li}_{x} \mathrm{CoO}_{2}$ [16] and $\mathrm{Li}_{x} \mathrm{NiO}_{2}$ [17].

The $\mu^{+}$SR spectra were measured at the surface muon beam line of the RIKEN-RAL Muon Facility at ISIS (U.K.) using a liquid-He flow-type cryostat in the $T$ range between 10 and $400 \mathrm{~K}$. The experimental techniques were described elsewhere [18]. $\chi$ was measured using a SQUID magnetometer (MPMS, Quantum Design) in the $T$ range between 5 and $200 \mathrm{~K}$ under a magnetic field of $H=100$ Oe.

Figure 1 shows the zero field (ZF) and longitudinal field (LF) $\mu^{+}$SR spectrum for the $\mathrm{Li}_{0.73} \mathrm{CoO}_{2}$ sample obtained at 100 and $225 \mathrm{~K}$. At $100 \mathrm{~K}$, the $\mathrm{ZF}$ spectrum exhibits a typical Kubo-Toyabe (KT) behavior with a minimum at $t \sim 6 \mu \mathrm{s}$, meaning that the implanted muons see the internal magnetic field $\left(H_{\text {int }}\right)$ due to the nuclear magnetic moments of ${ }^{7} \mathrm{Li},{ }^{6} \mathrm{Li}$, and ${ }^{59} \mathrm{Co}$. The applied $\mathrm{LF}$ clearly reduces the relaxation rate, i.e., the time slope, by decoupling $H_{\text {int }}$. Although the ZF spectrum still shows KT behavior at $225 \mathrm{~K}$, the relaxation rate is smaller than at $100 \mathrm{~K}$.

In order to estimate the KT parameters precisely, the ZF and two LF spectra were fitted simultaneously by a combination of a dynamic Gaussian KT function $\left[G^{\operatorname{DGKT}}\left(\Delta, \nu, t, H_{\mathrm{LF}}\right)\right]$ and a background (BG) signal from
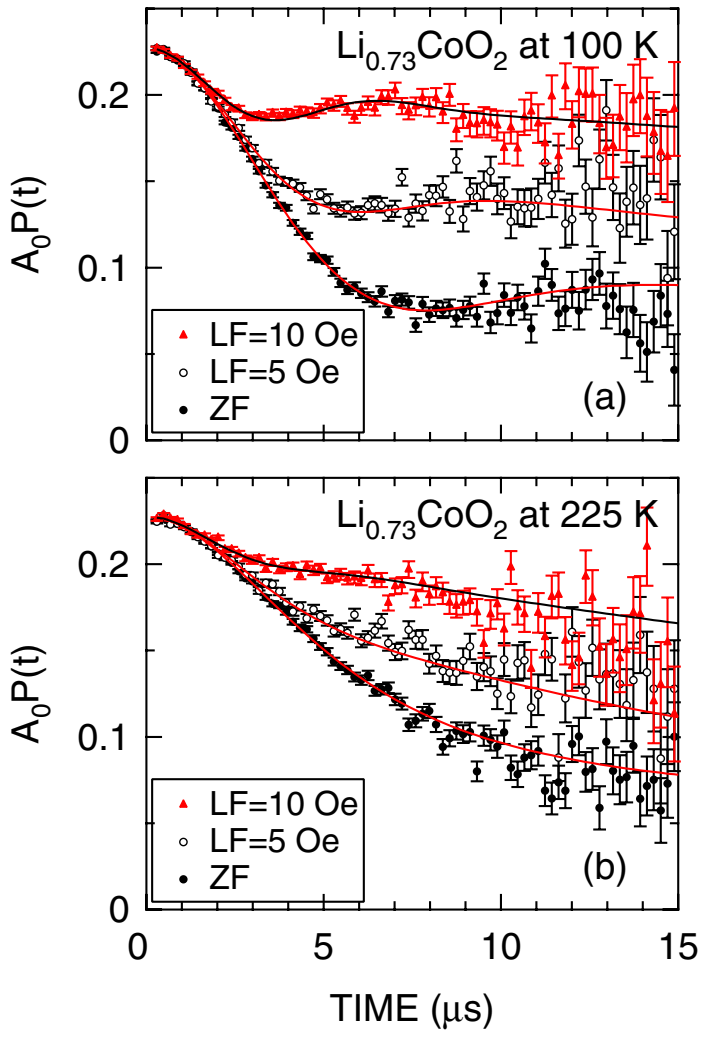

FIG. 1 (color online). ZF and two LF $\mu^{+}$SR spectra for $\mathrm{Li}_{0.73} \mathrm{CoO}_{2}$ measured at (a) $100 \mathrm{~K}$ and (b) $225 \mathrm{~K}$. Solid lines represent the fit result using Eq. (1). the fraction of muons stopped mainly in the sample holder, which is made of high-purity aluminum;

$$
A_{0} P_{\mathrm{LF}}(t)=A_{\mathrm{KT}} G^{\mathrm{DGKT}}\left(\Delta, \nu, t, H_{\mathrm{LF}}\right)+A_{\mathrm{BG}},
$$

where $A_{0}$ is the initial $(t=0)$ asymmetry, $A_{\mathrm{KT}}$ and $A_{\mathrm{BG}}$ are the asymmetries associated with the two signals. $\Delta$ is the static width of the local field distribution at the disordered sites, and $\nu$ is the field fluctuation rate. When $\nu=0$ and $H_{\mathrm{LF}}=0, G^{\mathrm{DGKT}}\left(t, \Delta, \nu, H_{\mathrm{LF}}\right)$ is the static Gaussian $\mathrm{KT}$ function $G_{z z}^{\mathrm{KT}}(t, \Delta)$ in ZF. At first, we fitted all the ZF spectra using common $A_{\mathrm{KT}}$ and $A_{\mathrm{BG}}$ in Eq. (1). The "global fit" provided that $A_{\mathrm{KT}}=0.16406 \pm 0.00002(0.1689 \pm$ $0.0002)$ and $A_{\mathrm{BG}}=0.06350 \pm 0.00002 \quad(0.0692 \pm$ 0.0002) for $\mathrm{Li}_{0.73} \mathrm{CoO}_{2}\left(\mathrm{Li}_{0.53} \mathrm{CoO}_{2}\right)$. Then, using the obtained $A_{\mathrm{KT}}$ and $A_{\mathrm{BG}}$, one $\mathrm{ZF}$ and two LF spectra were global-fitted using common $\Delta$ and $\nu$ at each $T$.

Figure 2 shows the $T$ dependencies of both $\Delta$ and $\nu$ for the two samples together with $\chi$ measured in a 100 Oe magnetic field. For $\mathrm{Li}_{0.73} \mathrm{CoO}_{2}, \Delta$ is almost constant for $5 \leq T \leq 250 \mathrm{~K}$, indicating that the $\mu^{+}$are most probably stable in the crystal lattice until $\sim 300 \mathrm{~K} . \Delta$ then rapidly decreases, but levels off again for $T \geq 325 \mathrm{~K}$. The $\nu(T)$ curve is almost $T$-independent up to $150 \mathrm{~K}$, starts to increase at $\sim 150 \mathrm{~K}$, and exhibits a maximum at $275 \mathrm{~K}$. Above $275 \mathrm{~K}, \nu$ decreases to $0.7 \times 10^{6} \mathrm{~s}^{-1}$ at $\sim 325 \mathrm{~K}$, and finally becomes almost $T$-independent above $350 \mathrm{~K}$. The increase in $\nu$ between 150 and $275 \mathrm{~K}$ is well explained by a thermal activation process [Figs. $2(\mathrm{~g})$ and $2(\mathrm{~h})$ ], which signals the onset of diffusive motion of either $\mathrm{Li}^{+}$or $\mu^{+}$ above $150 \mathrm{~K}$. The clear decrease in $\Delta$ at $\sim 300 \mathrm{~K}$ also suggests an additional diffusion of $\mathrm{Li}^{+}$or $\mu^{+}$.

The $\chi(T)$ curve exhibits a small anomaly around $150 \mathrm{~K}$ with a thermal hysteresis of $\sim 10 \mathrm{~K}$, while there is no indication of any magnetic anomalies in the $T$ range between 200 and $300 \mathrm{~K}$ [Figs. 2(e) and 2(f)] [15,16]. This suggests that the change in the $\mu^{+} \mathrm{SR}$ parameters around $150 \mathrm{~K}$ is caused by an intrinsic change in $\mathrm{Li}_{x} \mathrm{CoO}_{2}$, but the change around $300 \mathrm{~K}$ is visible only by $\mu^{+} \mathrm{SR}$. The increase in $\nu$ above $150 \mathrm{~K}$ is, thus, most unlikely due to $\mu^{+}$ diffusion, but it is rather due to $\mathrm{Li}^{+}$diffusion; i.e., either a freezing of the $\mathrm{Li}^{+}$motion or an order-disorder transition of the $\mathrm{Li}+$ ions occurs below around $150 \mathrm{~K}$. This is also supported by a recent ${ }^{7} \mathrm{Li}$ NMR experiment [19], in which the NMR linewidth-versus- $T$ curve for $\mathrm{Li}_{0.6} \mathrm{CoO}_{2}$ exhibits a steplike decrease with $T$ around $150 \mathrm{~K}$ by motional narrowing due to $\mathrm{Li}^{+}$diffusion. Since such diffusion naturally increases a local structural symmetry, it is reasonable that $\Delta$ slightly decreases with $T$ around $150 \mathrm{~K}$. On the other hand, both $\mathrm{Li}^{+}$and $\mu^{+}$are inferred to be diffusing above $300 \mathrm{~K}$, resulting in the large decrease in $\Delta$ caused by motional narrowing. Actually, because $\Delta \leq 0.1 \nu$ above $300 \mathrm{~K}$, Eq. (1) is roughly equivalent to an exponential relaxation function $[\exp (-\lambda t)]$, and it is difficult to estimate $\Delta$ and $\nu$ precisely at high $T$.

The result for the $\mathrm{Li}_{0.53} \mathrm{CoO}_{2}$ sample is very similar to that of $\mathrm{Li}_{0.73} \mathrm{CoO}_{2}$, although $\Delta_{T \rightarrow 0}\left(\mathrm{Li}_{0.53} \mathrm{CoO}_{2}\right)<$ $\Delta_{T \rightarrow 0}\left(\mathrm{Li}_{0.73} \mathrm{CoO}_{2}\right)$ due to the decrease in the number den- 

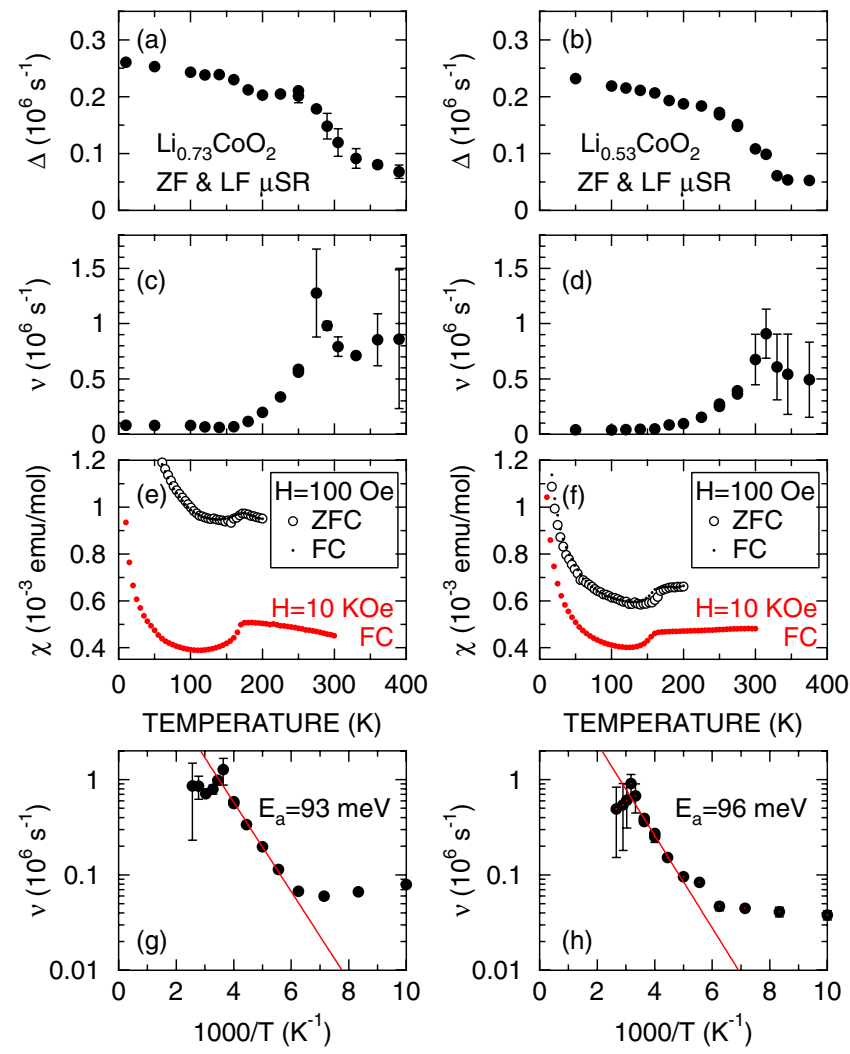

FIG. 2 (color online). For $\mathrm{Li}_{0.73} \mathrm{CoO}_{2}$ and $\mathrm{Li}_{0.53} \mathrm{CoO}_{2}$, respectively, we show $T$ dependences of (a),(b) field distribution width $(\Delta),(\mathrm{c}),(\mathrm{d})$ field fluctuation rate $(\nu),(\mathrm{e}),(\mathrm{f})$ susceptibility $(\chi)$, and $(\mathrm{g}),(\mathrm{h})$ the $\log (\nu)$-vs- $1 / T$ curve. $\Delta$ and $\nu$ were obtained by fitting both ZF and LF spectra using Eq. (1). $\chi$ was measured in both field cooling (FC) and zero field cooling (ZFC) mode with $H=100$ Oe. In (e),(f), the $\chi$ data [16] measured in FC mode with $H=10 \mathrm{kOe}$ for $\mathrm{Li}_{0.75} \mathrm{CoO}_{2}$ and $\mathrm{Li}_{0.52} \mathrm{CoO}_{2}$ were also plotted for comparison. The straight lines in (g) and (h) show the activated diffusive behavior discussed in the text.

sity of $\mathrm{Li}^{+}$ions, as reported previously $[15,16]$. Also, the magnitude of $\nu$ of $\mathrm{Li}_{0.53} \mathrm{CoO}_{2}$ is smaller than $\nu$ of $\mathrm{Li}_{0.73} \mathrm{CoO}_{2}$ in the whole $T$ range measured, but the $\nu(T)$ curve for both samples shows a clear increase with $T$ above $150 \mathrm{~K}$ and a maximum around $300 \mathrm{~K}$.

In order to predict the muon site(s) and to confirm the reliability of the above assumption that $\mathrm{Li}^{+}$ions diffuse above $150 \mathrm{~K}$ whereas $\mu^{+}$diffuse only above $300 \mathrm{~K}$, we performed electrostatic potential $\left(\phi_{E}\right)$ calculations for the $\mathrm{Li}_{x} \mathrm{CoO}_{2}$ lattice using a point-charge model and the program DIPELEC [20]. As seen in Fig. 3, the site in the vicinity of the $\mathrm{O}^{2-}$ ions is more stable for $\mu^{+}$than the site in the $\mathrm{Li}$ plane for the whole $x$ range between 1 and 0 . This means that $\mu^{+}$'s are bound to the $\mathrm{O}^{2-}$ ions so as to make a stable $\mu^{+}-\mathrm{O}^{2-}$ bond in $\mathrm{Li}_{x} \mathrm{CoO}_{2}$. This is a common situation in oxides, as, for example, in the case for the high- $T_{c}$ cuprates [21]. Since $\mu^{+}$'s are assigned as an ideal point charge, such a $\mu^{+}-\mathrm{O}^{2-}$ bond should be purely ionic. In fact, dipole field calculations for the site in the vicinity of the $\mathrm{O}^{2-}$ ions provide that $\Delta_{\text {calc }}=0.43 \times 10^{6} \mathrm{~s}^{-1}\left(0.35 \times 10^{6} \mathrm{~s}^{-1}\right)$ for
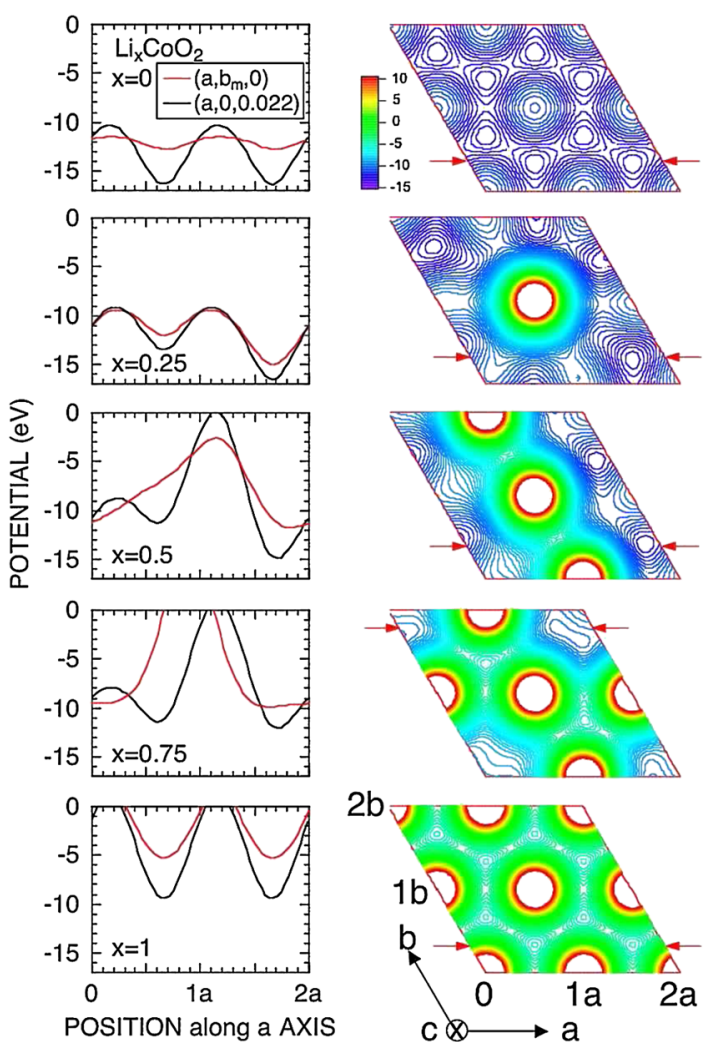

FIG. 3 (color online). The variation of electrostatic potential ( $\phi_{E}$ ) along the $a$ axis at $b=b_{m}$ and $c=0$ (on the Li plane) and $b=0$ and $c=0.022$ ( $1 \AA$ away from the $\mathrm{O}^{2-}$ ions) in the hexagonal lattice of $\mathrm{Li}_{x} \mathrm{CoO}_{2}$ with $x=0,0.25,0.5,0.75$, and 1 (from top to bottom). Here, $b_{m}$ denotes the $b$, at which electrostatic potential exhibits a minimum. The right-hand panels show the corresponding distribution of $\phi_{E}$ in the Li plane. Arrows on the $b$ axis in the right-hand panels represents $b_{m}$.

$\mathrm{Li}_{x} \mathrm{CoO}_{2}$ with $x=3 / 4(1 / 2)$. Furthermore, $\Delta_{\text {calc }}$ is found to be comparable to $\Delta$ measured at low $T$ in the whole $x$ range for $\mathrm{Li}_{x} \mathrm{CoO}_{2}$ [16], if we consider the reduction of $\Delta$ by the electric field gradient effect on the nuclear moments with $I \geq 1[22,23]$. This suggests that the point-charge model is acceptable for determining the muon site(s) in $\mathrm{Li}_{x} \mathrm{CoO}_{2}$. As a result, it is clarified that, as $T$ increases from $5 \mathrm{~K}$, the $\mathrm{Li}^{+}$ions start to diffuse above $150 \mathrm{~K}\left(=T_{d}^{\mathrm{Li}}\right)$ and then the $\mu^{+}$diffuse above $300 \mathrm{~K}\left(=T_{d}^{\mu}\right)$, in spite of the mass difference between $\mu^{+}$and $\mathrm{Li}^{+}\left(m_{\mathrm{Li}^{+}} / m_{\mu^{+}} \sim 63\right)$ because the muons form a hydrogenlike bond with oxygen.

Finally, we estimate $D_{\mathrm{Li}}$ using the obtained fluctuation rate $\nu$ as directly measuring the jump rate. Figure 4(a) shows the possible jump paths for the Li ions, i.e., the direct jump to the nearest (vacant) Li site (path number 1) and the jump to the interstitial site in the center of the oxygen tetrahedron (path number 2). Assuming that $\nu$ corresponds to the jump rate of the $\mathrm{Li}$ ions between the neighboring sites, $D_{\mathrm{Li}}$ is given by [24]

$$
D_{\mathrm{Li}}=\sum_{i=1}^{n} \frac{1}{N_{i}} Z_{v, i} s_{i}^{2} \nu
$$



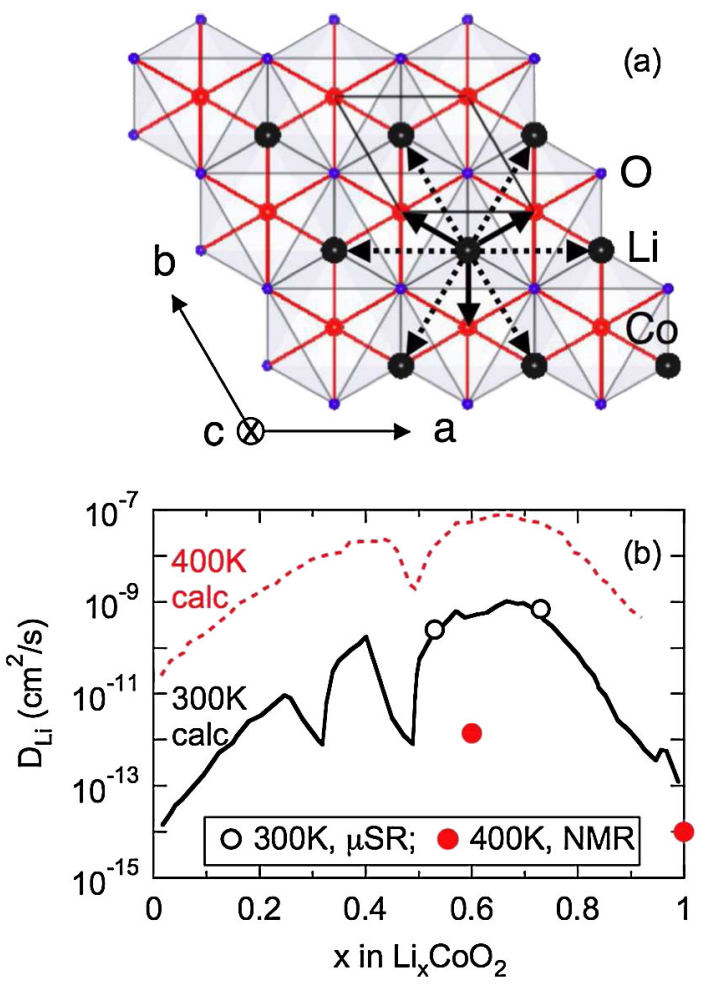

FIG. 4 (color online). (a) Possible jump paths for Li ions. Broken arrows represent the direct jump to nearest (vacant) Li site (path number 1), whereas solid arrows represent the jump to an interstitial site in the center of the oxygen tetrahedron (path number 2). (b) The relationship between $D_{\mathrm{Li}}$ and $x$ in $\mathrm{Li}_{x} \mathrm{CoO}_{2}$ as extracted from our $\mu^{+}$SR experiment (open circles). The solid line and the dashed line represent the predictions by firstprinciples calculations [5] at $T=300$ and $400 \mathrm{~K}$, respectively, when the effective vibration frequency is a typical value $\left(10^{13} \mathrm{~s}^{-1}\right)$. Sharp minima in the predicted curve (at $x=1 / 3$ and $1 / 2$ ) are caused by $\mathrm{Li}$ ordering. The ${ }^{7} \mathrm{Li} \mathrm{NMR}$ result $[4,19]$ for $T=400 \mathrm{~K}$ is also plotted (solid dot) for comparison.

where $N_{i}$ is the number of Li sites in the $i$ th path, $Z_{v, i}$ is the vacancy fraction, and $s_{i}$ is the jump distance. Here, we naturally restrict the path to lie in the $c$ plane, i.e., along the $2 \mathrm{D}$ channel, because it is most unlikely that the $\mathrm{Li}$ ions jump across the $\mathrm{CoO}_{2}$ plane to an adjacent $\mathrm{Li}$ plane. Therefore, $N_{1}=6, N_{2}=3, s_{1}$ is equivalent to the $a$-axis length, $s_{2}=a / \sqrt{3}, Z_{v, 1}=0.27$ for $\mathrm{Li}_{0.73} \mathrm{CoO}_{2}(0.47$ for $\left.\mathrm{Li}_{0.53} \mathrm{CoO}_{2}\right)$, and $Z_{v, 2}=1$. As a result, we obtain $D_{\mathrm{Li}}=$ $(7 \pm 2) \times 10^{-10} \mathrm{~cm}^{2} / \mathrm{s}\left[(2.5 \pm 0.8) \times 10^{-10} \mathrm{~cm}^{2} / \mathrm{s}\right]$ for $\mathrm{Li}_{0.73} \mathrm{CoO}_{2}\left[\mathrm{Li}_{0.53} \mathrm{CoO}_{2}\right]$ at $300 \mathrm{~K}$. Here, $\nu(300 \mathrm{~K})$ for $\mathrm{Li}_{0.73} \mathrm{CoO}_{2}$ was estimated from the extrapolation of the linear relationship between $\log [\nu]$ and $T^{-1}$ [see Fig. 2(g)]. The estimated $D_{\mathrm{Li}}$ is found to be very consistent with the prediction by first-principles calculations [5], as seen in Fig. 4(b). Note that the jump paths used in Eq. (2) are the same as those for the first-principles calculations. This means that there is no ambiguous factor for estimating $D_{\mathrm{Li}}$ by $\mu^{+} \mathrm{SR}$. Since $\mu^{+} \mathrm{SR}$ detects $\nu$ ranging from $\sim 0.01 \Delta$ to $\sim 10 \Delta$, it is applicable for materials with $D_{\mathrm{Li}}=$
$10^{-12}-10^{-9} \mathrm{~cm}^{2} / \mathrm{s}$, when $N=10, Z_{v}=1, s=1 \mathrm{~nm}$, and $\Delta=0.1 \times 10^{6} \mathrm{~s}^{-1}$.

In conclusion, we have been able to determine the $\mathrm{Li}$ diffusion coefficient, $D_{\mathrm{Li}}$, of $\mathrm{Li}_{x} \mathrm{CoO}_{2}$ from the fluctuation rate of the field experienced by the muons in interaction with the nuclear moments of the diffusing ions. The value was found to be in good agreement with theoretical predictions. Consequently, we would like to suggest $\mu^{+}$SR as a novel probe to investigate $\mathrm{Li}$ diffusion, especially for materials containing transition metal ions.

This work was performed at the RIKEN-RAL Muon Facility at ISIS, and we thank the staff for help with the $\mu^{+}$SR experiments. We appreciate T. Ohzuku and K. Ariyoshi for sample preparation and K. Yoshimura for discussion. J. S. and Y. I. are supported by the KEK-MSL Inter-University Program for Overseas Muon Facilities. This work is also supported by Grant-in-Aid for Scientific Research (B), 19340107, MEXT, Japan.

*e0589@mosk.tytlabs.co.jp

†Present address: Muon Science Laboratory, Institute of Materials Structure Science, High Energy Accelerator Research Organization, Tsukuba, Japan.

[1] T. Ohzuku and R. Brodd, J. Power Sources 174, 449 (2007).

[2] I. Tomeno and M. Oguchi, J. Phys. Soc. Jpn. 67, 318 (1998).

[3] K. Nakamura et al., Solid State Ionics 121, 301 (1999).

[4] K. Nakamura et al., Solid State Ionics 135, 143 (2000).

[5] A. Van der Ven and G. Ceder, Electrochem. Solid-State Lett. 3, 301 (2000).

[6] K. Mizushima et al., Solid State Ionics 3-4, 171 (1981).

[7] C. Y. Yao et al., J. Power Sources 54, 491 (1995).

[8] S.-Il Pyun and Y.-M. Choi, J. Power Sources 68, 524 (1997).

[9] K. Dokko et al., J. Electrochem. Soc. 148, A422 (2001).

[10] K. Dokko et al., J. Power Sources 189, 783 (2009).

[11] K. A. Striebel et al., J. Electrochem. Soc. 143, 1821 (1996).

[12] Y.H. Rho and K. Kanamura, J. Electrochem. Soc. 151, A1406 (2004).

[13] H. Xia et al., J. Electrochem. Soc. 154, A337 (2007).

[14] S. B. Tang et al., J. Alloys Compd. 449, 300 (2008).

[15] J. Sugiyama et al., Phys. Rev. B 72, 144424 (2005).

[16] K. Mukai et al., Phys. Rev. Lett. 99, 087601 (2007).

[17] J. Sugiyama et al., Phys. Rev. B 78, 144412 (2008).

[18] G. M. Kalvius et al., in Handbook on the Physics and Chemistry of Rare Earths, edited by K. A. Gschneidner et al. (Elsevier Science, Amsterdam, 2001), Vol. 32, Ch. 206, and references cited therein.

[19] K. Nakamura et al., Solid State Ionics 177, 821 (2006).

[20] K. M. Kojima et al., Phys. Rev. B 70, 094402 (2004).

[21] T. R. Adams et al., Hyperfine Interact. 86, 561 (1994).

[22] R. S. Hayano et al., Phys. Rev. B 20, 850 (1979).

[23] C. T. Kaiser et al., Phys. Rev. B 62, R9236 (2000).

[24] R. J. Borg and G. J. Dienes, An Introduction to Solid State Diffusion (Academic, San Diego, 1988). 